\title{
Fermi Level Control of Gold Nanoparticle by the Support: Activation of the Catalysis for Selective Aerobic Oxidation of Alcohols *
}

\author{
Shin-ichi Naya, ${ }^{a}$ Miwako Teranishi, ${ }^{a}$ Ryo, Aoki ${ }^{b}$ and Hiroaki Tada*a,b \\ a Environmental Research Laboratory, Kinki University, 3-4-1, Kowakae, \\ Higashi-Osaka, Osaka 577-8502, Japan. \\ ${ }^{\mathrm{b}}$ Department of Applied Chemistry, Faculty of Science and Engineering, \\ Kinki University, 3-4-1, Kowakae, Higashi-Osaka, Osaka 577-8502, Japan. \\ * To whom correspondence should be addressed: TEL: +81-6-6721-2332, FAX: +81-6-6727-2024, \\ E-mail: h-tada@apch.kindai.ac.jp.
}


Table S1. Surface area, particle size, supplier, and isoelectric point (IEP) of metal oxide support.

\begin{tabular}{ccccc}
\hline Support & $\begin{array}{c}\text { surface area } \\
/ \mathrm{m}^{2} \mathrm{~g}^{-1}\end{array}$ & $\begin{array}{c}\text { particle size } \\
/ \mathrm{nm}\end{array}$ & supplier & IEP \\
\hline $\mathrm{SrTiO}_{3}$ & 20 & 58 & Aldrich & 2.4 \\
$\mathrm{ZnO}$ & $15-25$ & $50-70$ & Aldrich & 9.8 \\
$\mathrm{TiO}_{2}$ & 8.1 & 150 & Ishihara Sangyo & 6.0 \\
$\mathrm{BiVO}_{4}$ & 0.54 & 1200 & --- & 2.5 \\
$\mathrm{WO}_{3}$ & 11.8 & 71 & Aldrich & 1.5 \\
\hline
\end{tabular}

Table S2. Au loading amount, Au particle size $d$, standard deviation $\sigma$, number of count and heating temperature $T_{\mathrm{c}}$ and time $t_{\mathrm{c}}$.

\begin{tabular}{lcrrrrr}
\hline catalyst & Au mass $\%$ & $d / \mathrm{nm}$ & $\sigma / \mathrm{nm}$ & count & $T_{\mathrm{c}} / \mathrm{K}$ & $t_{\mathrm{c}} / \mathrm{h}$ \\
\hline $\mathrm{Au} / \mathrm{SrTiO}_{3}$ & 0.62 & 2.7 & 0.6 & 128 & 673 & 4 \\
$\mathrm{Au} / \mathrm{SrTiO}_{3}$ & 0.61 & 3.9 & 1.2 & 111 & 773 & 4 \\
$\mathrm{Au} / \mathrm{SrTiO}_{3}$ & 0.63 & 8.4 & 1.9 & 133 & 873 & 24 \\
$\mathrm{Au} / \mathrm{ZnO}$ & 0.31 & 2.9 & 0.5 & 109 & 673 & 4 \\
$\mathrm{Au} / \mathrm{TiO}_{2}$ & 0.24 & 2.1 & 0.3 & 102 & 673 & 1 \\
$\mathrm{Au} / \mathrm{TiO}_{2}$ & 0.24 & 2.9 & 1.1 & 105 & 873 & 2 \\
$\mathrm{Au} / \mathrm{TiO}_{2}$ & 0.24 & 3.5 & 0.4 & 130 & 773 & 4 \\
$\mathrm{Au} / \mathrm{TiO}_{2}$ & 0.25 & 7.7 & 1.1 & 105 & 873 & 4 \\
$\mathrm{Au} / \mathrm{TiO}_{2}$ & 0.25 & 12.5 & 1.6 & 107 & 873 & 24 \\
$\mathrm{Au} / \mathrm{BiVO}_{4}$ & 0.30 & 16.1 & 3.9 & 106 & 873 & 4 \\
$\mathrm{Au} / \mathrm{WO}_{3}$ & 0.07 & 11.1 & 2.1 & 88 & 873 & 4 \\
\hline
\end{tabular}


(a)

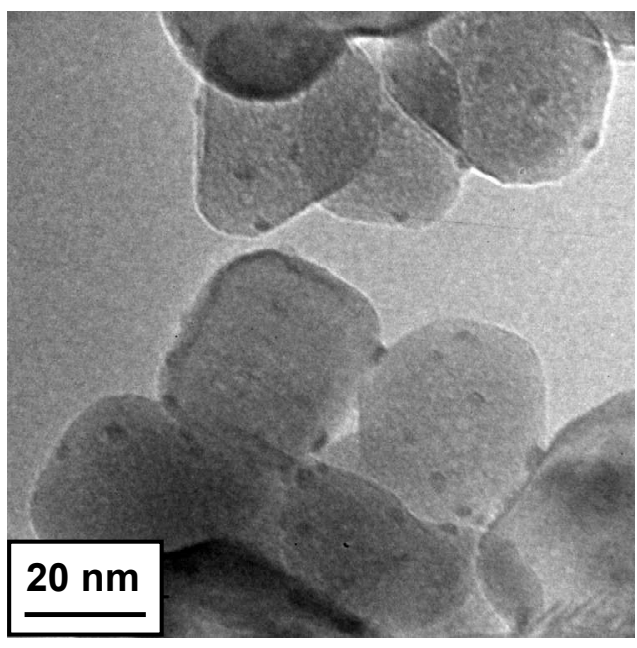

(b)

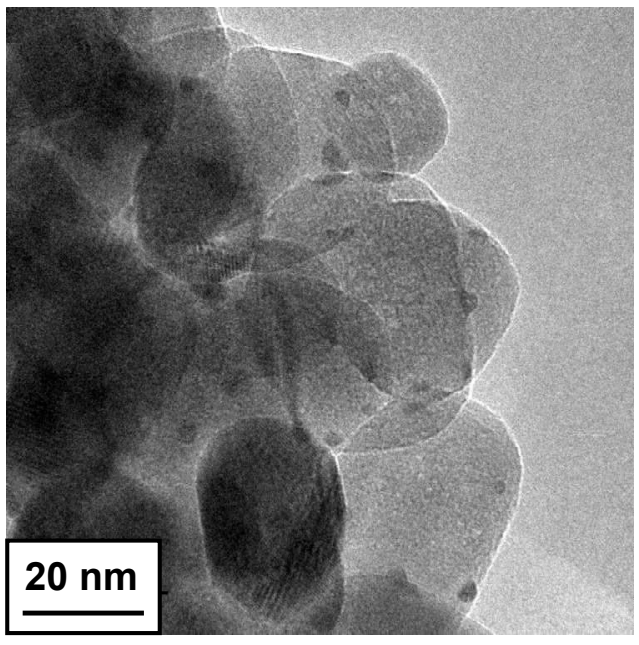

Figure S1.TEM image of $\mathrm{Au} / \mathrm{SrTiO}_{3}\left(d_{\mathrm{Au}}=3.3 \mathrm{~nm}\right)$ before (a) and after (b) alcohol-oxidation for $24 \mathrm{~h}$. 